\title{
OPINIÃO DOS AGENTES COMUNITÁRIOS DE SAÚDE SOBRE O USO DA VIDEOCONFERÊNCIA NA CAPACITAÇÃO EM SAÚDE AUDITIVA INFANTIL
}

\section{Community health agents' opinions on the use of videoconference in children health hearing training}

\author{
Tatiana Mendes de Melo ${ }^{(1)}$, Kátia de Freitas Alvarenga ${ }^{(2)}$, \\ Wanderléia Quinhoneiro Blasca ${ }^{(3)}$, Marcel Frederico de Lima Taga ${ }^{(4)}$
}

\begin{abstract}
RESUMO
Objetivo: avaliar a videoconferência como ferramenta instrucional na capacitação dos agentes comunitários de saúde, em saúde auditiva infantil, sob a perspectiva destes profissionais. Método: a casuística foi composta por 50 agentes comunitários de saúde, divididos aleatoriamente em dois grupos de acordo com a metodologia de ensino: 31 profissionais participaram da capacitação de forma presencial e 19 por meio de videoconferência. A atividade ocorreu em um encontro de oito horas, por meio de aulas expositivas e utilizando material didático adaptado e validado para o português proposto pela Organização Mundial da Saúde de forma simultânea para ambos os grupos. A avaliação da videoconferência como ferramenta instrucional foi realizada por meio de questionário elaborado para o presente estudo. Resultados: a avaliação da videoconferência foi muito semelhante em ambos os grupos, sendo que a ferramenta de ensino foi considerada como de fácil entendimento e permitindo a interação com o ministrante. Conclusão: a videoconferência foi uma ferramenta válida e aceita pelos profissionais estudados para ser utilizada em programas de capacitação em saúde auditiva infantil.
\end{abstract}

DESCRITORES: Videoconferência; Capacitação; Saúde da Família; Perda Auditiva

\section{INTRODUÇÃO}

Em 1994, o Ministério da Saúde assumiu a Estratégia Saúde da Família (ESF) como forma de reorganização do modelo tradicional de cuidados à saúde em nível nacional, a partir das experiências

(1) Fonoaudióloga; Docente do curso de Fonoaudiologia da Universidade de Guarulhos, Guarulhos, São Paulo, Brasil; Mestre em Fonoaudiologia.

(2) Fonoaudióloga; Professora Associada do Departamento de Fonoaudiologia da Faculdade de Odontologia de Bauru Universidade de São Paulo, FOB-USP, Bauru, São Paulo, Brasi; Doutora em Distúrbios da Comunicação Humana.

(3) Fonoaudióloga; Professora do Departamento de Fonoaudiologia da Faculdade de Odontologia de Bauru - Universidade de São Paulo, FOB-USP, Bauru, São Paulo, Brasil; Doutora em Distúrbios da Comunicação Humana.

(4) Matemático; Estatístico do Hospital de Reabilitação de Anomalias Craniofaciais - Universidade de São Paulo, HRAC-USP, Bauru, São Paulo, Brasil.

Conflito de interesses: inexistente bem sucedidas do Programa de Agentes Comunitários de Saúde (PACS), que contribuiu para a redução das mortalidades infantil e materna nas regiões Norte e Nordeste.

Um dos componentes fundamentais para a reorientação qualitativa desejada refere-se à existência de profissionais preparados para atuar na perspectiva da atenção integral à saúde, em atividades que contemplem desde ações de promoção até reabilitação e capazes de uma prática multiprofissional e interdisciplinar ${ }^{1}$. Neste sentido, em 2004, o Ministério da Saúde propôs a Política Nacional de Edu cação Permanente em Saúde, como recurso operacional para o desenvolvimento de habilidades dos profissionais de Saúde da Família, em substituição à proposta dos Pólos de Capacitação, Formação e Educação Permanente de Recursos Humanos para a Saúde da Família ${ }^{2}$.

A educação permanente possibilita a atualização e aperfeiçoamento profissional, proporcionando uma forma de assistência humanizada, de 
alta qualidade e com resolubilidade impactante não somente frente à demanda da população como também em relação aos custos do Sistema Único de Saúde ${ }^{2-3}$.

Atualmente, o movimento de ampliação das atividades de capacitação utilizando os recursos tecnológicos da informática, especialmente para os profissionais que atuam em regiões de difícil acesso ou geograficamente distantes dos principais centros urbanos é crescente, principalmente no contexto internacional. Neste sentido, a videoconferência destaca-se como uma ferramenta tecnológica importante para facilitar o acesso desses à atualização de conhecimentos.

$A$ videoconferência como instrumento frente às atividades de educação permanente em saúde é recente no país ${ }^{4-5}$, mas experiências internacionais acumuladas até o presente momento evidenciaram não somente resultados positivos, com a mudança do comportamento do profissional após a capacitação, como também grande aceitação do recurso tecnológico para estas atividades ${ }^{6-19}$.

$\mathrm{Na}$ Portaria SAS n'. 587 de 2004, a capacitação das equipes de atenção básica, por meio do apoio técnico das equipes dos serviços de saúde auditiva na Média e Alta complexidade, é uma normativa ${ }^{20}$. De acordo com resultados apresentados ${ }^{21}$, a capacitação de agentes comunitários de saúde (ACS), em saúde auditiva infantil, proporciona uma mudança positiva na atuação dos mesmos, no atendimento junto à população, preocupando-se mais com o desenvolvimento da audição e linguagem da criança.

Considerando a importância da capacitação dos ACS em saúde auditiva infantil, bem como o uso de ferramentas para otimizar o aperfeiçoamento destes profissionais, o objetivo do presente estudo foi avaliar a opinião dos ACS sobre o uso da videoconferência como ferramenta instrucional na capacitação destes profissionais na área de saúde auditiva infantil.

\section{MÉTODO}

Todos os ACS das equipes Saúde da Família e do PACS do município de Bauru foram convidados a participar de um curso de capacitação sobre saúde auditiva infantil. Inicialmente, foi entregue a ficha de inscrição da atividade que deveria ser devolvida pelo ACS no dia de realização da mesma. Esta ficha, contendo questionamentos referentes à idade, escolaridade, informações relacionadas à maternidade/paternidade, e tempo de atuação como ACS, foi preenchida pelos participantes para que, posteriormente, a caracterização dos grupos estudados pudesse ser efetuada.
Após a entrega dessa ficha, cada participante recebeu o material da capacitação, contendo a apostila, uma caneta, um bloco de notas e um crachá com um número específico. Este procedimento foi adotado a fim de preservar a identificação dos mesmos no momento da aplicação do questionário aplicado para verificar a opinião dos ACS sobre a capacitação por videoconferência.

Os profissionais foram divididos em dois grupos, de acordo com a metodologia empregada na atividade de educação permanente, isto é, 31 ACS tomaram parte da capacitação sobre saúde auditiva infantil de forma presencial e 19, por meio de videoconferência, correspondendo ao total da capacidade da sala. A seleção dos participantes dos dois grupos foi realizada de maneira aleatória, no momento da entrega do material gráfico.

A forma de abordagem da capacitação se deu por meio de aulas expositivas, com intervalo final para perguntas. A atividade aconteceu em um dia útil, ou seja, esteve inserida na rotina de trabalho dos ACS e ocorreu em um encontro de oito horas, com intervalos previamente programados.

O material didático utilizado constituiu em uma apostila da Organização Mundial da Saúde (OMS) - Primary ear and hearing care training resource basic, intermediate and advanced levels, adaptado e validado para o português ${ }^{21}$. A adoção deste material permitiu que os ACS acompanhassem as atividades realizadas de forma interativa e desenvolvendo determinadas atividades em conjunto com o ministrante. Também foram empregados vídeos e arquivos de áudio para exemplificar situações vivenciadas pelos indivíduos deficientes auditivos.

A capacitação que se deu de forma presencial e a que ocorreu a distância realizaram-se simultaneamente, por meio de videoconferência, a fim de garantir que o mesmo conteúdo fosse ministrado aos dois grupos de estudo com concomitância. Para o acompanhamento da capacitação na sala remota, um tutor esteve presente, a fim de conduzir os questionamentos elaborados pelo grupo pesquisa para os palestrantes, além de propiciar o monitoramento/feedback sobre o áudio e vídeo utilizados. 0 tutor também responsabilizou-se pela entrega/recebimento dos questionários utilizados para avaliação da videoconferência como ferramenta de ensino.

No início do curso os participantes que realizaram a capacitação a distância foram orientados sobre o desenvolvimento da aula expositiva e, caso houvesse alguma dúvida no transcorrer desta, poderiam interromper a qualquer momento, pois, por meio do equipamento de videoconferência, era possível a comunicação entre os grupos e o ministrante do curso. 
Como dinâmica de ensino, após tópicos préestabelecidos, foi fornecido um tempo de interação e discussão entre os ACS das duas salas, os quais ofereceram exemplos de situações vividas em suas visitas domiciliares, como também esclarecimentos de possíveis dúvidas.

Para a avaliação da videoconferência como ferramenta instrucional na capacitação de ACS foi elaborado um questionário, contendo perguntas voltadas à dinâmica da atividade, em relação à aquisição de conhecimento, interatividade e tempo disponível, assim como em relação ao material didático empregado e a qualidade de transmissão do áudio e vídeo utilizados. Este questionário foi aplicado ao final do curso, para ambos os grupos, a fim de verificar se o tipo de capacitação, presencial ou por meio de videoconferência, poderia influenciar na qualidade da formação e na satisfação dos participantes.

Este estudo foi aprovado pelo Comitê de Ética em Pesquisa em Seres Humanos da Faculdade de Odontologia de Bauru, da Universidade de São
Paulo, sob protocolo número 007/2007 e autorizado pela Secretaria Municipal da Saúde de Bauru.

Os dados do estudo foram armazenados em banco de dados do EXCEL e foi realizada a análise descritiva, por meio dos resultados, em porcentagem, em cada questão do questionário. Para análise comparativa dos grupos em relação à idade e ao tempo de atuação como ACS, utilizou-se o teste $T$ de Student. Para a comparação em relação às informações referentes à maternidade/paternidade e ao bairro de atuação dos ACS foram utilizados os testes qui-quadrado e Fisher, respectivamente.

\section{RESULTADOS}

A atividade de educação permanente foi realizada com $100 \%$ de participação dos ACS do município de Bauru. A Tabela 1 apresenta a comparação entre os grupos em relação às variáveis: idade, maternidade/paternidade, bairro e tempo de atuação como ACS.

Tabela 1 - Análise inferencial das variáveis estudadas, em relação ao tipo de capacitação realizada

\begin{tabular}{ccc}
\hline Variável & $\mathbf{P}$ & Teste estatístico \\
\hline Idade & 0,526 & T de Student \\
Tempo de atuação & 0,754 & T de Student \\
Filhos & 0,980 & Qui-quadrado \\
Bairro de atuação & 0,106 & Exato de Fisher \\
\hline
\end{tabular}

A formação acadêmica dos ACS caracterizouse, na sua maioria, pelo ensino médio em ambos os grupos (26 ACS no grupo controle e 18 no grupo da capacitação por videoconferência). O restante dos profissionais que receberam a atividade a distância apresentava ensino fundamental. Adicionalmente, no grupo controle havia três ACS com ensino fundamental e dois com ensino superior.

A distribuição das respostas obtidas nas questões do questionário de avaliação da videoconferência como ferramenta educacional na capacitação em ambos os grupos pode ser vista na Tabela 2.

Os resultados da avaliação da videoconferência como ferramenta instrucional foi bastante semelhante em ambos os grupos. Os profissionais referiram que o conteúdo foi de fácil entendimento (questão três) e houve interação com o ministrante (questão quatro). Os aspectos relacionados ao áudio e vídeo também foram considerados satisfatórios (questões cinco e seis).

Dos profissionais envolvidos nesta atividade, apenas um ACS já havia participado de programas de capacitação por meio da videoconferência (questão 10). Quando questionados se havia interesse em participar de outros cursos por meio da videoconferência (questão 11), todos os participantes do estudo foram unânimes e relataram o interesse em realizar outras atividades de educação permanente utilizando este tipo de ferramenta.

\section{DISCUSSÃO}

Ao considerar que a competência profissional dos recursos humanos em saúde é um dos componentes para a reorientação do modelo assistencial e que em 2008, o Brasil apresenta 28 mil equipes de Saúde da Família, conferindo cobertura a 87 milhões de brasileiros ${ }^{22}$, torna-se necessário 0 incentivo à incorporação dos recursos tecnológicos para promoção de atividades de educação permanente desses profissionais, de forma igualitária em todo território nacional.

A videoconferência como ferramenta para disseminação das atividades de capacitação de 
Tabela 2 - Distribuição das respostas obtidas no questionário de avaliação da videoconferência como ferramenta educacional

\begin{tabular}{|c|c|c|c|c|c|c|c|}
\hline \multicolumn{8}{|c|}{ QUESTIONÁRIO } \\
\hline Questão & Capacitação & Não & (\%) & Sim & (\%) & Total & (\%) \\
\hline \multirow{2}{*}{$\begin{array}{l}\text { Você acha que o programa de } \\
\text { capacitação utilizado } \\
\text { (videoconferência e material } \\
\text { impresso) foi um recurso } \\
\text { didático que proporcionou o } \\
\text { maior entendimento? }\end{array}$} & Presencial & & & 30 & $(100)$ & 30 & $(100)$ \\
\hline & Videoconferência & & & 19 & $(100)$ & 19 & (100) \\
\hline \multirow{2}{*}{$\begin{array}{l}\text { O programa proposto } \\
\text { proporcionou o interesse? }\end{array}$} & Presencial & & & 30 & $(100)$ & 30 & (100) \\
\hline & Videoconferência & & & 19 & $(100)$ & 19 & $(100)$ \\
\hline \multirow{2}{*}{$\begin{array}{l}\text { Achou que as aulas por } \\
\text { videoconferência foram de fácil } \\
\text { entendimento? }\end{array}$} & Presencial & & & 30 & $(100)$ & 30 & (100) \\
\hline & Videoconferência & & & 19 & $(100)$ & 19 & $(100)$ \\
\hline \multirow{2}{*}{$\begin{array}{l}\text { Achou que a aula por } \\
\text { videoconferência foi interativa? }\end{array}$} & Presencial & & & 30 & $(100)$ & 30 & (100) \\
\hline & Videoconferência & 1 & $(5)$ & 18 & $(95)$ & 19 & $(100)$ \\
\hline \multirow{2}{*}{ A transmissão foi adequada? } & Presencial & & & 30 & $(100)$ & 30 & $(100)$ \\
\hline & Videoconferência & & & 19 & $(100)$ & 19 & (100) \\
\hline \multirow{2}{*}{$\begin{array}{l}\text { O som da apresentação estava } \\
\text { adequado? }\end{array}$} & Presencial & & & 30 & $(100)$ & 30 & $(100)$ \\
\hline & Videoconferência & 2 & (11) & 17 & (89) & 19 & $(100)$ \\
\hline \multirow{2}{*}{$\begin{array}{l}\text { O tempo de apresentação foi } \\
\text { satisfatório? }\end{array}$} & Presencial & & & 30 & $(100)$ & 30 & (100) \\
\hline & Videoconferência & & & 19 & $(100)$ & 19 & (100) \\
\hline \multirow{2}{*}{$\begin{array}{l}\text { Você mudaria as imagens } \\
\text { utilizadas? }\end{array}$} & Presencial & 30 & $(100)$ & & & 30 & (100) \\
\hline & Videoconferência & 18 & $(95)$ & 1 & (5) & 19 & (100) \\
\hline \multirow{2}{*}{$\begin{array}{l}\text { Você mudaria a forma de } \\
\text { apresentação dos textos? }\end{array}$} & Presencial & 30 & $(100)$ & & & 30 & (100) \\
\hline & Videoconferência & 19 & $(100)$ & & & 19 & $(100)$ \\
\hline \multirow{2}{*}{$\begin{array}{l}\text { Você já havia participado de um } \\
\text { programa de capacitação } \\
\text { utilizando outras tecnologias na } \\
\text { educação (videoconferência)? }\end{array}$} & Presencial & 29 & $(97)$ & 1 & (3) & 30 & (100) \\
\hline & Videoconferência & 19 & $(100)$ & & & 19 & (100) \\
\hline \multirow{2}{*}{$\begin{array}{l}\text { Você participaria novamente de } \\
\text { outro curso utilizando a } \\
\text { videoconferência? }\end{array}$} & Presencial & & & 30 & $(100)$ & 30 & $(100)$ \\
\hline & Videoconferência & & & 19 & $(100)$ & 19 & (100) \\
\hline
\end{tabular}

profissionais da saúde é amplamente utilizada internacionalmente ${ }^{6-19}$, mas no Brasil, estudos nesta área são escassos ${ }^{4-5}$, uma vez que somente em 2006 o Ministério da Saúde instituiu a Comissão Permanente em Telessaúde, que é responsável pelo desenvolvimento de trabalhos com vistas à estruturação de atividades de educação permanente envolvendo os recursos tecnológicos, tais como a videoconferência ${ }^{23}$.

Neste sentido, o presente estudo que enfocou a avaliação da videoconferência para capacitação dos ACS na área de saúde auditiva infantil, sob a ótica destes profissionais, é particularmente oportuno, pois vai ao encontro da visão atual do Ministério da Saúde, que vem assumindo ações que irão auxiliar a equipe da Saúde da Família a realizar assistência integral e com qualidade à população em todo o país ${ }^{24}$.

No âmbito da Política de Atenção à Saúde Auditiva, a videoconferência pode proporcionar a disseminação do conhecimento desta área nas regiões mais distantes do Brasil e aperfeiçoar a atuação profissional dos ACS, e demais profissionais da Saúde da Família, nas ações de promoção e prevenção das alterações auditivas na população até o apoio e suporte às famílias dos portadores de deficiência auditiva ${ }^{5}$.

A avaliação dos ACS sobre o uso da videoconferência na capacitação em saúde auditiva foi positiva, independente do tipo de capacitação realizada, 
e permeada pela qualidade adequada, durante toda a atividade, dos aspectos elencados a seguir.

Primeiramente, a aceitação deste tipo de tecnologia é decorrente da interação, mais próxima à realidade de um curso presencial, proporcionada pela videoconferência ${ }^{25}$. A qualidade do áudio/ vídeo e adequação da conexão são fatores que também interferem na opinião dos participantes quanto à utilização da videoconferência nestas atividades ${ }^{9-13}$.

Outro fator importante foi a organização cuidadosa da atividade que envolveu a seleção adequada dos conteúdos, a utilização do material gráfico interativo e coerente aos conteúdos ministrados na capacitação, o recurso de aulas expositivas divididas em módulos com intervalos para perguntas, 0 uso de imagens e vídeos para ilustrar os conceitos a serem adquiridos e a presença do tutor na sala que recebeu o curso de educação permanente foram fatores que provavelmente também contribuíram para o êxito da atividade ${ }^{26,27}$.
Como apresentado anteriormente, dos profissionais envolvidos nesta atividade, apenas um ACS já havia participado de programas de capacitação por meio da videoconferência, o que confirma que o uso desta tecnologia, até o presente momento, é pouco difundido para propiciar a educação permanente dos profissionais de saúde no país.

\section{CONCLUSÃO}

Desta maneira, foi possível concluir que a videoconferência foi uma ferramenta válida e aceita pelos ACS para ser utilizada em programas de capacitação na área de saúde auditiva infantil.

\section{AGRADECIMENTOS}

À Secretaria Municipal de Saúde do município de Bauru, São Paulo, pela participação e apoio efetivo dos gestores para a realização da pesquisa.

\begin{abstract}
Purpose: to check the opinion from community health agents about the use of videoconference as instructional tool in children hearing health program training. Method: the group was made up by 50 community health agents divided randomly in two groups, with the following sample: 31 community health agents by face-to-face instruction and 19 , by videoconference. The training was provided in eight hours, through classes and a textbook translated and adapted into the Brazilian Portuguese language proposed by World Health Organization, in both groups, simultaneously. In order to investigate the opinion about the use of videoconference as instructional tool in training program, a questionnaire was done and applied, in both group, after the course. Results: the videoconference quality evaluation , compared by face-to-face instruction, in the opinion of health care professionals was similar, and was considered easy and interactive. Conclusion: the videoconference was a valid and accepted learning tool to be adopted in training programs for community health agents.
\end{abstract}

KEYWORDS: Videoconferencing; Training; Family Health; Hearing Loss

\section{REFERÊNCIAS}

1. Gil CRR. Formação de recursos humanos em saúde da família: paradoxos e perspectivas. Cad Saude Publica. 2005;21(2):490-8.

2. Ceccim RB. Educação Permanente em Saúde: descentralização e disseminação de capacidade pedagógica na saúde. Cien Saude Colet. 2005;10(4):975-86.

3. Fontinele Júnior K. Programa saúde da família PSF comentado. Goiânia: AB Editora; 2003.

4. Godoy S; Nogueira MS; Hayashida M; Mendes IAC. Administração de injetáveis por via intramuscular na região ventroglútea: avaliação após treinamento por videoconferência. Rev RENE. 2003;4(1):86-92.

5. Melo TM, Alvarenga KF, Blasca EQ, Taga MFL. Capacitação de agentes comunitários de saúde em saúde auditiva: efetividade da videoconferência. Pró-Fono Rev de Atual Cient. 2010;22(2):139-44.

6. Hackett J, Madden DL, Viney KA, Naylor CJ. Evaluation of three population health capacity building projects delivered by videoconferencing in NSW. N S W Public Health Bull. 2009;20(11-12):182-6.

7. Church EA, Heath OJ, Curran VR, Bethune C, Callanan TS, Cornish PA. Rural 
professionals' perceptions of interprofessional continuing education in mental health. Health Soc Care Community. 2010 May 27. [Epub ahead of print].

8. O'Regan K, Marsden P, Sayers G, Morrissey M, Hegarty $H$, Allen $M$ et al. Videoconferencing of a national program for residents on evidence-based practice: early performance evaluation. J Am Coll Radiol. 2010;7(2):138-45.

9. Spitzer RF, Kives S, Ornstein M, Caccia N, Stephens D, Flood C, et al. Videoconferencing for resident teaching of subspecialty topics: the pediatric and adolescent gynecology experience at the Hospital for Sick Children. J Pediatr Adolesc Gynecol. 2008;21(6):343-6.

10. Miller PA, Huijbregts M, French E, Taylor D, Reinikka K, Berezny L et al. Videoconferencing a stroke assessment training workshop:effectiveness, acceptability, and cost. J Contin Educ Health Prof. 2008;28(4):256-69.

11. Rossaro L, Tran TP, Ransibrahmanakul K, Rainwater JA, Csik G, Cole SL et al. Hepatitis C videoconferencing: the impact on continuing medical education for rural healthcare providers. Telemed $\mathrm{J}$ E Health. 2007;13(3):269-77.

12. Augestad KM, Lindsetmo RO. Overcoming distance: video-conferencing as a clinical and educational tool among surgeons. World J Surg. 2009;33(7):1356-65.

13. Doarn CR. The power of video conferencing in surgical practice and education. World J Surg. 2009;33(7):1366-7.

14. Bahaadinbeigy $\mathrm{K}$, Yogesan $\mathrm{K}$, Wootton $\mathrm{R}$. A survey of the state of telemedicine in Western Australia. J Telemed Telecare. 2010;16(4):176-80. 15. Durrani $H$, Khoja S. A systematic review of the use of telehealth in Asian countries. J Telemed Telecare. 2009;15(4):175-81.

16. Weeks DL, Molsberry DM. Pediatric advanced life support re-training by videoconferencing compared to face-to-face instruction: a planned non-inferiority trial. Resuscitation. 2008;79(1):109-17.

17. Karlinsky H; Dunn C; Clifford B; Atkins J; Pachev G; Cunningham $\mathrm{K}$ et al. Workplace injury management: using new technology to deliver and

http://dx.doi.org/10.1590/S1516-18462010005000134 RECEBIDO EM: 12/05/2010

ACEITO EM: 21/08/2010

Endereço para correspondência:

Tatiana Mendes de Melo

Rua Alto Garças, no 1176 - Cidade Patriarca

São Paulo - SP

CEP: 03546-000

E-mail: tati_usp@yahoo.com.br evaluate physician continuing medical education. $\mathrm{J}$ Occup Rehabil.2006;16:719-30.

18. Metter DF; Woolley FR; Bradley Y; Morrison J; Phillips WT; Salman U et al. Teaching radiology residenct didactics using videoconferencing. Acad Radiol. 2006;13:1276-85.

19. Ahn HH; Kim JE; Ko NY; Seo SH; Kim SN; Kye YC. Videoconferencing journal club for dermatology residency training: an attitude survey. Acta Derm Venérelo. 2007;87:397-400.

20. Brasil. Secretaria de Assistência a Saúde. Ministério da Saúde. Portaria № 587, 07 de outubro de 2004. Que determina que as Secretarias de Estado da Saúde dos estados adotem as providências necessárias à organização e implantação das Redes Estaduais de Atenção à Saúde Auditiva e de outras providências. Diário Oficial da República Federativa do Brasil. p. 105, 11 de outubro, 2004.

21. Alvarenga KF; Bevilacqua MC; Martinez MANS; Melo TM; Blasca WQ; Taga MFL. Proposta para capacitação de agentes comunitários de saúde em saúde auditiva. Pro Fono. 2008; 20(3):171-6.

22. Brasil. Ministério da Saúde. SecretariaExecutiva. Mais saúde: direito de todos: 2008 - 2011. 2. ed. - Brasília: Editora do Ministério da Saúde, 2008. 100 p.

23. Brasil. Portaria no 561, de 16 de março de 2006. Institui, no âmbito do Ministério da Saúde, a Comissão Permanente de Telessaúde. Diário Oficial da União. 17 mar 2006; Seção 1:59.

24. Swanepoeli DW, Clark JL, Koekemonr D, Hall JW, Krumm M, Ferrari DV et al. Telehealth in audiology: The need and potential to reach underserved communities. International Journal of Audiology 2010; 49: 195-202.

25. Vargas MRM. Educação a distância e as novas tecnologias: o uso da videoconferência em treinamentos organizacionais. 2002. [texto na internet]. Acesso em: 2007 ago 16. Disponível em: http://www.abed.org.br/publique/cgi/cgilua.exe/sys/ start.htm? UserActiveTemplate=1 por\&from $\% 5$ Finfo $\% 5$ Findex $=6$ \&infoid $=29 \&$ query $=$ simple \&search $\% 5$ Fby\%5Fauthorname=all\&search $\% 5 F$ by $\% 5 F f i e l d=t$ ax\&search $\% 5 \mathrm{Fby} \% 5 \mathrm{Fheadline}=$ false\&search $\% 5 \mathrm{~F}$ by $\% 5$ Fkeywords=any\&search $\% 5 \mathrm{Fby} \% 5 \mathrm{Fpriority}=$ all\&search $\% 5 \mathrm{Fby} \% 5 \mathrm{Fsection}=\mathrm{all} \&$ search $\% 5 \mathrm{Fby} \%$ 5 Fstate=all\&search $\% 5$ Ftext $\% 5$ Foptions $=$ all \&sid= 48\&text=videoconfer\%EAncia\&tpl=printerview.

26. Birden H, Page S. Teaching by videoconference: a commentary on best practice for rural education in health professionals. Rural Remote Health. 2005;5(2):356.

27. Moehr JR, Anglin CR, Schaafsma JP, Pantazi SV, Anglin S, Grimm NA. Videoconferencing based health: its implications for health promotion and health care. Methods Inf Med. 2005;44(2):334-41. 